Research Article

\title{
Image Restoration Based on Gradual Reweighted Regularization and Low Rank prior
}

\author{
Fengling Wang (iD \\ College of Arts Management, Shandong University of Arts, Jinan 250300, China \\ Correspondence should be addressed to Fengling Wang; flwangart@163.com
}

Received 6 August 2019; Revised 29 February 2020; Accepted 10 March 2020; Published 22 April 2020

Academic Editor: Akemi Gálvez

Copyright (c) 2020 Fengling Wang. This is an open access article distributed under the Creative Commons Attribution License, which permits unrestricted use, distribution, and reproduction in any medium, provided the original work is properly cited.

\begin{abstract}
Digital restoration of image with missing data is a basic need for visual communication and industrial applications. In this paper, making full use of priors of low rank and nonlocal self-similarity a gradual reweighted regularization is proposed for matrix completion and image restoration. Sparsity-promoting regularization produces much sparser representation of grouped nonlocal similar blocks of image by solving a nonconvex minimization problem. Moreover, an alternation direction method of multipliers algorithm is developed to speed up iterative solving of the above problem. Image block classification further enhances the adaptivity of the proposed method. Experiments on simulated matrix and natural image show that the proposed method obtains better image restoration results, where most lost information is reorcovered and few artifacts are produced.
\end{abstract}

\section{Introduction}

In today's information age, the rapid development of computer technology facilitates digital record of rich visual information in pictures and videos, etc. However, data loss and incomplete information of image due to damage and bad transmission bring negative impact on the communication and research of visual media [1-3]. In this paper, digital image restoration and enhancement are addressed to restore missed and damaged part in digital images using residual information, imaging context and prior knowledge of image, in order to make the restored image close to its original one as much as possible [4-9]. Digital restoration of degraded images provides possibility of rapid communication, appreciation, and research for people from different fields $[3,4,10]$.

As a fundamental ill-posed inverse problem in image processing and low-level vision, digital image restoration aims to reconstruct a latent high-quality image from its degraded observation $[7,11]$. Up to now, image restoration technology has made great progress, and many advanced methods have been introduced based on a variety of optimization models, mainly including variational calculus and partial differential equations $[4,5,8,12]$, methods based on such priors as exemplar matching and synthesis [13-15], sparse representation and low rank approximation [16-26], etc.

Image restoration methods based on variational calculus and partial differential equations propagate/diffuse local structure information from the external of missing areas to the internal based on smoothness prior [4]. Multiple variants use different models (linear, nonlinear, isotropic, or anisotropic) to extend the information in a given particular propagation direction or taking into account some geometric information such as the curvature of local neighborhood pixels $[4,5,12]$. However, a large number of experiments show that these methods have some limitations: although successful for images with piecewise smooth structures or small gaps, they are not suitable for textured images, especially when missing area is larger. In order to preserve edges, after a few of iterations, they can easily lead to excessive smoothness and blur in the repaired areas [7].

In order to restore damaged areas of textured structures, on the basis of pioneering works of texture synthesis [27, 28], another image restoration method is put forward based on exemplar matching and synthesis. For an exemplar needed to be repaired, a texture repair method tries to find the best 
matching sample to it in a certain neighborhood and restores missing information by sampling or copying corresponding pixels for exemplar synthesis [13-15]. If enough similar candidate blocks are found in the image or in an outside image data base, a better image restoration can be achieved. Recently current researches of this kind mainly focus on the multiscale refinement of exemplar matching and synthesis, improvement in distance measure used to find matching blocks, faster searching method of matching blocks, optimized block processing ordering, and filling into unknown pixels from the matching blocks [7].

In the past twenty years, sparse representation theory of signal and image has been a hot research field in signal processing. With the vigorous development of sparse sampling and compressed sensing, sparse prior knowledge is introduced into image restoration algorithms $[16,17,21]$. Here, it is assumed that image is a sparse signal under a set of specific transformation bases (the sparsity of signal depends mainly on the given bases). These bases are composed of atoms stored in a dictionary matrix, which can be obtained by various dictionary learning methods [29]. Recently, as a second-order sparse prior, low rank matrix approximation is also introduced to recover a latent low rank matrix structure of image from its noisy observation. This research branch has attracted increasing attention in image processing due to its popularity and effectiveness [18, 30, 31]. For example, an efficient filtering algorithm is proposed in [20] to sparsely represent image patches using singular value decomposition (SVD) and to remove noise in image by iterative singular value shrinkage. Another algorithm proposed in [26] utilizes nonlocal selfsimilarity (NSS) and low rank approximation, which includes two steps of SVD based on a special hard thresholding. The method based on weighted nuclear norm minimization [24] assigns different weights to singular values such that a more reasonable soft thresholding is carried out, which has achieved excellent performance in different image processing tasks.

Natural images are usually rich in texture and complex in structure, and they are just of approximately low rank. In this paper, the nonlocal self-similarity is used to gather similar image blocks, and a nonconvex optimization problem with gradual reweighted regularization is proposed based on low rank property of the group matrix of similar blocks. By solving the minimization problem using an alternation direction method of multipliers (ADMM) algorithm, we present much sparser representation of image duo to the sparsity-promoting gradual regularization. The proposed method obtains better image restoration results, where most lost information is recovered and few artifacts are produced.

The remainder of the paper is organized as follows. Related works of low rank minimization and the gradual reweighted regularization for the restoration of matrix and image are introduced in Section 2, respectively. In Section 3, experimental results on matrix completion and image restoration demonstrate the merit of the proposed approaches compared to classic methods. Finally, we conclude this paper in Section 4.

\section{Gradual Reweighted Regularization}

2.1. Low Rank Minimization. Sparse representation and compressed sensing have achieved large success in image processing [29, 32]. Inspired by this idea low rank matrix approximation has drawn increasing attention and broadly interest in recent years, where rank is interpreted as a measure of second order sparsity (matrix) [33]. Aiming to recover an underlying low rank matrix from its degraded observation, low rank matrix approximation can robustly and efficiently handle high-dimensional data with high noise or severe corruption, due to the fact that many types of data (raw or after some nonlinear transforms) reside near single or multiple subspaces [34]. Singular value decomposition is often an effective approach to solve low rank model using special thresholding operations on the singular values of observation matrix [24, 31, 35-37].

In this paper, we consider image restoration as a problem of recovering a low rank signal matrix in which its entries are observed in the presence of lost information. More specifically, the objective is to recover an unknown matrix $X$ from its observed degraded data $Y=H X+\eta$, where $H$ is a degeneracy operator containing the spatial position of information loss in $Y$, and $\eta$ is a noise disruption (although we do not consider noise in the paper). The low rank matrix approximation estimates $X$ by solving the following nuclear norm minimizing problem with a $F$-norm data fidelity [31]:

$$
\min _{X} \lambda\|X\|_{*}+\|Y-H X\|_{F}^{2}
$$

where $\|X\|_{*}=\sum_{i} \sigma_{i}(X)=\|\sigma(X)\|_{1}$ is the nuclear norm of $X$, $\sigma(X)=\left(\sigma_{1}(X), \ldots, \sigma_{r}(X)\right) \quad\left(\sigma_{1}(X) \geq \cdots \geq \sigma_{r}(X) \geq 0\right) \quad$ is the vector of singular values of $X, r$ is the rank of $X$, and $\lambda$ is a positive constant.

When $H$ is an identity matrix, a solution of above problem can be obtained by [31].

$$
\widehat{X}=U \mathcal{S}_{\lambda}(\Sigma) V^{T},
$$

where $Y=U \Sigma V^{T}$ is the SVD of $Y$, and $\mathcal{S}_{\lambda}(\Sigma)$ is a soft thresholding function on the diagonal matrix $\Sigma$ :

$$
\mathcal{S}_{\lambda}(\Sigma)_{i i}=\max \left(\sigma_{i}(Y)-\frac{\lambda}{2}, 0\right)
$$

where $\sigma_{i}(Y)$ is the $i$-th singular value of $Y$.

2.2. Gradual Reweighted Regularization. In this paper, we employ an iteratively reweighted technology to recover matrix with the second-order sparsity (low rank) in the framework of sparsity-promoting low rank approximation [24, 38, 39]. To restore a degraded matrix $Y$ with pixels of missing information, we solve the following matrix completion model:

$$
\begin{array}{ll}
\min _{X} & \sum_{i} \omega_{i} \sigma_{i}(X) \\
\text { s.t. } & \mathscr{P}_{\Omega}(X)=\mathscr{P}_{\Omega}(Y),
\end{array}
$$

where $\omega_{i}$ is a nonnegative weight assigned to $\sigma_{i}(X)$ to emphasize the role of different singular values. $\Omega$ is a binary support indicator matrix with zeros indicating missing 
entries in the observed matrix $Y . \mathscr{P}$ is a projection operator: $\mathscr{P}_{\Omega}(Y)=\Omega \odot Y$, an element-wise Hardamard product of matrixes.

In the process of obtaining iteratively reweighted solution of the above-mentioned model in following algorithm, in order to obtain a sparser solution we adopt a weighting vector with gradual regularization:

$$
\omega_{i}^{l}=c\left(\sigma_{i}\left(X^{l-1}\right)+\epsilon^{l}\right)^{p / 2-1},
$$

where $\epsilon^{l} \in(0,1)$ is a gradual regularization sequence converging to zero, $c>0$ and $0 \leq p \leq 2$ are two constants, and $l$ is iteration steps.

In the strategy of gradual reweighted regularization (GRR), we first use a relatively large $\epsilon$ in (5), then we decrease it gradually in the iteration process as done in [39]. As shown in following experiments of matrix completion, we obtain sparser solutions and more exact results of image restoration. We think the success of the proposed method owes to that adopting a relatively large $\epsilon$ in the weights in foregoing iterations results in a weaker singular value shrinkage (see equation (3)) and more local minima being filled in the solution basin of the low rank model (4). In the iteration process, once the approximation solution enters into the correct basin, decreasing $\epsilon$ allows it to approach the optimal solution more closely as $\epsilon \longrightarrow 0$ [39]. The following Theorem 1 is also in favour of above argument.

We employ an alternation direction method of multipliers (ADMM) algorithm [40] to solve problem (4) by introducing a variable $E$ :

$$
\begin{array}{ll}
\min _{E, X, L} & \sum_{i} \omega_{i} \sigma_{i}(X)+\langle L, Y-X-E\rangle+\frac{\mu}{2}\|Y-X-E\|_{F}^{2} \\
\text { s.t. } & X+E=Y, \mathscr{P}_{\Omega}(E)=0
\end{array}
$$

where $L$ is a Lagrange multiplier and $\mu$ is a positive constant. We update $\{E, X, L\}$ sequentially by solving the following series of subproblems:

(1) Update of $E: X$ and $L$ are fixed, we solve

$$
\begin{aligned}
& E^{l}=\arg \min _{E}\left\|Y+L^{l-1} / \mu^{l-1}-X^{l-1}-E^{l-1}\right\|_{F}^{2} \\
& \text { s.t. }\left\|\mathscr{P}_{\Omega}\left(E^{l}\right)\right\|_{F}^{2}=0,
\end{aligned}
$$

Then, we have $E^{l}=Y+L^{l-1} / \mu^{l-1}-X^{l-1}$, s.t $\| \mathscr{P}_{\Omega}$ $\left(E^{l}\right) \|_{F}^{2}=0$.

(2) Update of $X: E$ and $L$ are fixed, we solve

$X^{l}=\arg \min _{X} \sum_{i} \omega_{i}^{l-1} \sigma_{i}\left(X^{l-1}\right)+\frac{\mu^{l-1}}{2}\left\|Y+\frac{L^{l-1}}{\mu^{l-1}}-X^{l-1}-E^{l}\right\|_{F}^{2}$.

Let $G^{l-1}=Y+L^{l-1} / \mu^{l-1}-E^{l}$, we first compute a singular value decomposition: $G^{l-1}=U \Sigma V^{T}$; then, we have $X^{l}=U \mathcal{S}_{\omega / \mu}(\Sigma) V^{T}$, where $\delta_{\omega / \mu}(\Sigma)_{i i}=$ $\max \left(\sigma_{i}\left(G^{l-1}\right)-\omega_{i}^{l-1} / \mu^{l-1}, 0\right)$.

(3) Update of $L$ : $E$ and $X$ are fixed, $L$ is easily obtained by

$$
L^{l}=L^{l-1}+\mu^{l-1}\left(Y-X^{l}-E^{l}\right) .
$$

The above optimization procedure is described in Algorithm 1.

Finally, we present a weak convergence result to ensure of a rational termination of Algorithm 1.

Theorem 1 (see [24]). If the weight sequence is arranged in a nondescending order, the sequence $\left\{X^{l}\right\}$ obtained by Algorithm 1 satisfies:

(1) $\lim _{l \longrightarrow \infty}\left\|X^{l+1}-X^{l}\right\|_{F}=0$,

(2) $\stackrel{l i m}{l \rightarrow \infty} \infty_{l \rightarrow}\left\|Y-E^{l}-X^{l}\right\|_{F}=0$.

2.3. Gradual Reweighted Regularization for Image Restoration. The nonlocal self-similarity (NSS) is widely used in image processing and computer vision $[20,24,26,35,37,41,42]$. There are many repeated local patterns across a natural image. After overlapping image blocks are extracted from a degraded image, for a given block with information loss, its nonlocal similar blocks can afford supplementary information for a better reconstruction of the degraded image. A simpler and effective grouping of mutually similar blocks can be realized by image block matching to find blocks that exhibit high correlation to a given one. The correlation between matrix rows and columns is naturally associated with the rank of matrix; thus, the formed group matrix by block matching is more likely to be of low rank than a whole image.

Considering the correlation between local image blocks, we propose an image restoration algorithm called as nonlocal gradual reweighted regularization method (NGRR), where the singular value thresholding driven by the gradual reweighted regularization provides highly sparser representation of image data. Because bigger singular values mainly describe image structures (major edge and texture information) $[24,43,44]$, while smaller singular values are mainly related to interference and noise, through shrinking of smaller singular values we can recover useful information of degraded image. The proposed method is an iterative reweighted filtering from image block estimation to pixel estimation based on a sparse low-rank prior, which includes extraction and classification of image blocks, block matching and grouping, singular value shrinkage filtering, aggregation, and iterative diffusion [37].

2.3.1. Block Extraction and Classification. In order to promote sparse low-rank representation of image data the nonlocal self-similarity of image blocks is used. According to image features image blocks are divided into two categories: block with texture or edge (contains rich structural information) and smooth block (single structure). Adaptive filtering of image blocks with different parameters can better recover the structure and detail of image. For simplicity, the standard deviation of local pixels is measured to distinguish between textured and smooth blocks. 


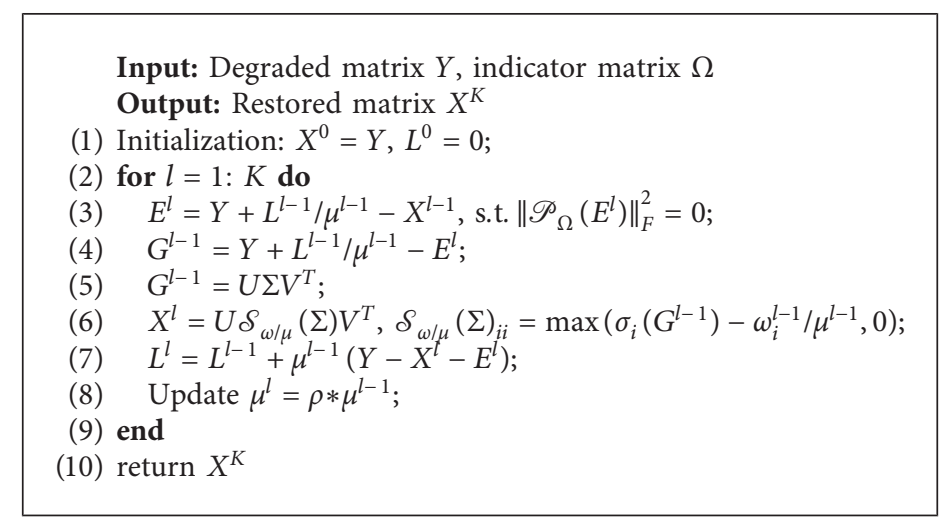

Algorithm 1: Gradual reweighted regularization for matrix completion.

2.3.2. Block Matching and Grouping. After overlapping, image blocks $\left\{y_{i}\right\}$ with size $W \times W$ are extracted from a degraded image $y$ and classified into textured or smooth blocks, and for each of image blocks $y_{i}$, block matching is carried out to assemble a group of similar blocks based on certain similarity criterion in a square $L \times L$ search window centered at $y_{i}$. The reference block $y_{i}$ and its $\left(N_{i}-1\right)$-most similar blocks denoted by $y_{j}\left(j=1,2, \ldots, N_{i}-1\right)$ are chosen to construct a group matrix $Y_{i}=\left[y_{i}, y_{1}, \ldots, y_{N_{i}-1}\right]$ using each of similar blocks as a column of it (see Figure 1). In the similar matrix $Y_{i}$, the corresponding columns from similar image blocks lead to lower rank of the group matrix $[24,35,44]$, and consequently, a highly sparse representation of image blocks is obtained by following singular value shrinkage filtering. For different type of target block $y_{i}$, when it is a texture or edge block, we select more similar blocks to it $\left(N_{i}\right.$ is bigger). When it is a flat block, we only need to select relatively few similar blocks to form a similar block matrix $\left(N_{i}\right.$ is smaller).

2.3.3. Singular Value Shrinkage Filtering. The singular value decomposition and shrinkage on above similar block matrix $Y_{i}$ is carried out in the iterative gradual reweighted regularization (Algorithm 1). Here, the singular energy of the similar matrix concentrates on a few of foregoing bigger singular values, which benefits the recovery of lost information from the observed image.

2.3.4. Aggregation. The proposed NGRR method first estimates latent image blocks; then it estimates each image pixel included in multiple image blocks to recover the whole image by aggregating estimated image blocks. Specifically, because overlapping image patches are extracted from a degraded image, a pixel belongs to multiple patches. Then, each pixel can be estimated by averaging multiple results from estimated patches.

2.3.5. Iterative Diffusion. For an image block located in the boundary of area with information loss, we can obtain a good estimate through above four steps. However, for image block located inside area with information loss, since there is no reliable information for similar block matching, the estimation based on above processing is not accurate. In order to solve this problem, we employ iteration diffusion to spread image information into the target area. That is to say, the previous output of image restoration is used as the initialization of next iteration. With the increase of number of iterations, image blocks located inside area with information loss will be gradually filled up with its surrounding information step by step.

The above image restoration procedure is described in Algorithm 2.

\section{Results and Analysis}

As a fundamental inverse problem in image processing and low-level vision, matrix completion and image restoration by filling in damaged area aim to reconstruct a plausible image from outside information of the damaged area. Below two experiments on matrix completion and image restoration are carried out to verify the rationality and effectiveness of the proposed method using the gradual regularization and low rank prior.

3.1. Matrix Completion. First, we compare the performance of the proposed method GRR with the state-of-the-art WNNM-MC method [24] in matrix completion.

In the following experiments, a synthetic low rank matrix $X$ is generated by the multiplication of two low rank matrixes: $X=A B^{T}$, where both $A$ and $B$ are of size $m \times r$. Each element of $A$ and $B$ is generated from a Gaussian distribution $\mathcal{N}(0,1), r=s_{r} \times m$ is used to constrain the rank of $X$. An observation matrix $Y$ is formed from the ground truth matrix $X$ by randomly dropping $s_{e} \times m^{2}$ entries of the matrix $X$. To be specific, in the generation process of the synthetic, low rank matrix $s_{r}$ is used to control the rank of the synthetic matrix, and $s_{e}$ is used to control its lost entries. We set $m=400$ and $c=400$. Let $s_{e}=0.1,0.2,0.3$, respectively, and $s_{r}$ vary from 0.01 to 0.5 with step length 0.01 . Initialize $\epsilon^{l}$ to be 1 , and update it down at a rate of $10 \%$ at each step. For each set of parameters $\left\{s_{r}, s_{e}\right\}$, we generate ten sets of data as test matrixes, and the performance of each method is measured by the mean value of ten sets of results. 


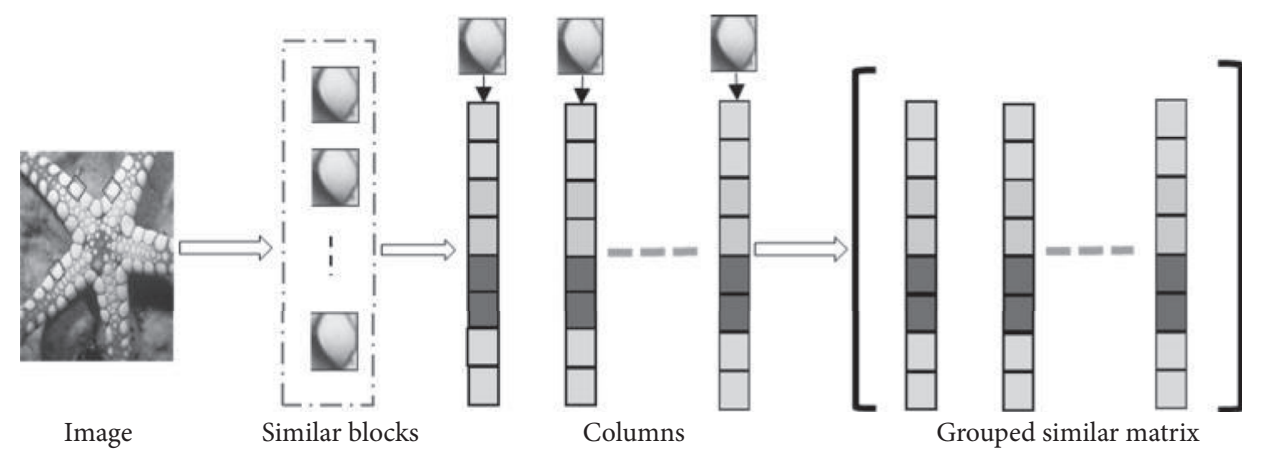

Figure 1: Block matching and grouping in NGRR.

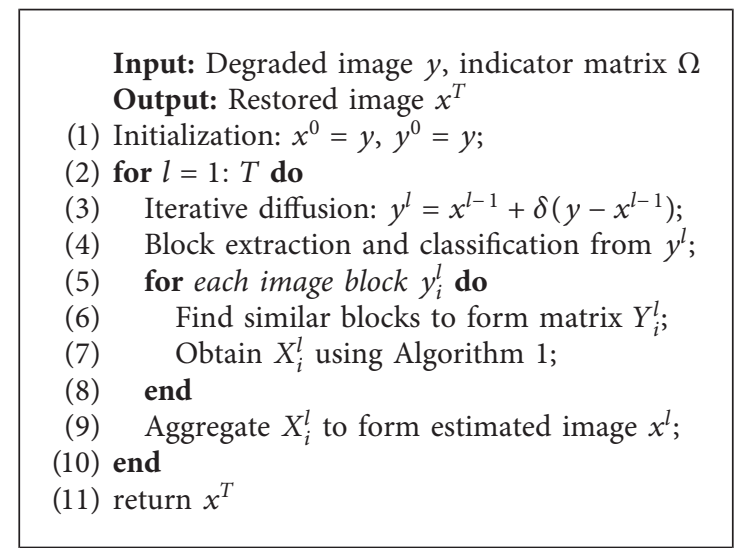

Algorithm 2: Gradual reweighted regularization for image restoration.

Experimental results are listed in Tables 1-4 for easy comparison.

By comparing relative errors listed in Tables 1-4 one can see that when $p=0$ and $\epsilon^{l}$ is a sequence approaching to zero, the relative errors of low-rank matrix recovery by $\mathrm{GRR}_{0}\left(\mathrm{GRR}_{p}\right.$ with $p=0$ ) are the smallest, especially when the matrix rank is bigger, where differences between relative errors are much more obvious. Thus, we adopt $p=0$ in (5) in following experiments of image restoration. At the same time, it is clear that the errors of the results by GRR are much smaller than that of WNNM-MC in all cases, which means that GRR has much better capability of low rank matrix reconstruction.

3.2. Image Restoration. In order to verify the performance of the proposed method in image restoration, we apply it to various natural images in different tasks: restoration of images with random loss of pixels. The proposed method (NGRR) is verified on test images shown in Figure 2 by comparing it with some classic image restoration methods, including BPFA [45], TNNR [46], two-stage low rank approximation (TSLRA) [26], and WNNM-MC [24]. All methods are implemented using MATLAB programming with gray scale range from 0 to 255 . In comparison of different image restoration methods, default parameter setting suggested by the authors in each method is made as in the source codes of these recovery algorithms from public websites.
In order to evaluate the quality of image recovery, we use peak signal-to-noise ratio (PSNR) and feature similarity (FSIM) [47] as quantitative measure indices. Although PSNR is sometimes inconsistent with human visual perception, it is widely used as a standard quantitative evaluation index of image quality and is closely related to visual perception. Further, based on the fact that human visual system understands image mainly according to its low-level features, FSIM combines phase consistency feature with gradient amplitude to measure the similarity between two images [47]. Larger PSNR and FSIM values mean higher quality of image recovery. The mathematical formulations of PSNR and FSIM are defined as follows:

$$
\begin{aligned}
& \text { PSNR }=10 \log _{10}\left(\frac{255^{2}}{(1 / N) \sum_{j=1}^{N}\left(x_{j}-\hat{x}_{j}\right)^{2}}\right), \\
& \mathrm{FSIM}=\frac{\sum_{j} S_{L, j} \cdot \mathrm{PC}_{m, j}}{\sum_{j} \mathrm{PC}_{m, j}},
\end{aligned}
$$

where $x_{j}$ and $\hat{x}_{j}$ are real image and its estimated one at pixel $j$, respectively; $S_{L, j}$ measures the similarity between real and estimated images, and $\mathrm{PC}_{m, j}$ represents the maximum of two phase consistency indices [47].

In order to better demonstrate advantages of the proposed NGRR method in following comparative experiments, 
TABLE 1: Relative errors of matrix recovery results by $\operatorname{GRR}_{p}\left(s_{e}=0.3, s_{r}=0.1: 0.05: 0.4\right)$.

\begin{tabular}{lccccccc}
\hline Rank & 40 & 60 & 80 & 100 & 120 & 140 \\
\hline$p=0$ & $5.20 E-11$ & $4.53 E-11$ & $4.51 E-11$ & $4.98 E-11$ & $1.08 E-10$ & $1.61 E-10$ & $2.77 E-10$ \\
$p=1$ & $1.10 E-07$ & $1.63 E-07$ & $1.40 E-07$ & $1.67 E-07$ & $3.43 E-07$ & $5.97 E-07$ & $1.07 E-06$ \\
$p=2$ & $1.24 E-07$ & $1.23 E-07$ & $1.94 E-07$ & $2.98 E-07$ & $6.42 E-02$ & $1.70 E-01$ & $2.50 E-01$ \\
\hline
\end{tabular}

TABLE 2: Relative errors of matrix recovery results by $\operatorname{GRR}_{0}\left(s_{e}=0.1, s_{r}=0.1: 0.05: 0.4\right)$.

\begin{tabular}{lccccccc}
\hline Rank & 40 & 60 & 80 & 100 & 120 & 140 \\
\hline WNNM-MC $_{\mathrm{GRR}_{0}}$ & $5.70 E-08$ & $1.14 E-07$ & $1.21 E-07$ & $1.58 E-07$ & $1.82 E-07$ & $2.09 E-07$ & $2.70 E-07$ \\
\hline
\end{tabular}

TABLE 3: Relative errors of matrix recovery results by $\operatorname{GRR}_{0}\left(s_{e}=0.2, s_{r}=0.1: 0.05: 0.4\right)$.

\begin{tabular}{lccccccc}
\hline Rank & 40 & 60 & 80 & 100 & 120 & 140 \\
\hline WNNM-MC & $9.12 E-08$ & $1.37 E-07$ & $1.68 E-07$ & $2.07 E-07$ & $2.60 E-07$ & $3.45 E-07$ & $4.53 E-07$ \\
GRR $_{0}$ & $4.53 E-11$ & $3.74 E-11$ & $3.64 E-11$ & $3.69 E-11$ & $3.65 E-11$ & $4.57 E-11$ & $1.06 E-10$ \\
\hline
\end{tabular}

TABLE 4: Relative errors of matrix recovery results by $\operatorname{GRR}_{0}\left(s_{e}=0.3, s_{r}=0.1: 0.05: 0.4\right)$.

\begin{tabular}{lccccccc}
\hline Rank & 40 & 60 & 80 & 100 & 120 & 140 \\
\hline WNNM-MC $_{\text {GRR }}$ & $1.22 E-07$ & $1.63 E-07$ & $2.23 E-07$ & $2.84 E-07$ & $3.82 E-07$ & $5.72 E-07$ & $1.85 E-06$ \\
\hline
\end{tabular}

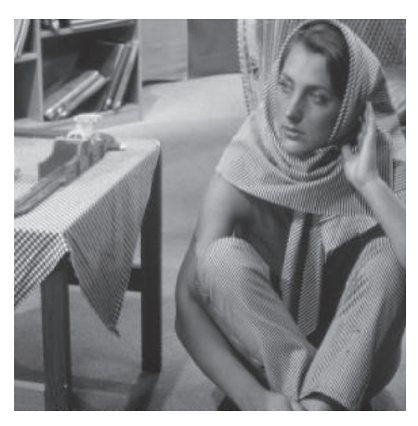

(a)

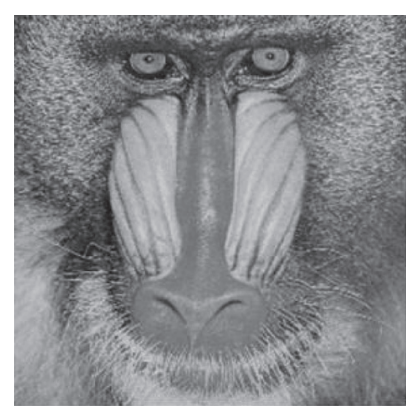

(d)

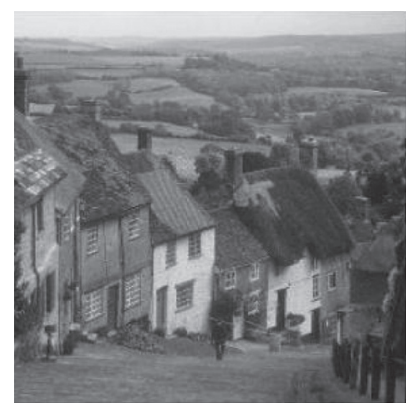

(g)

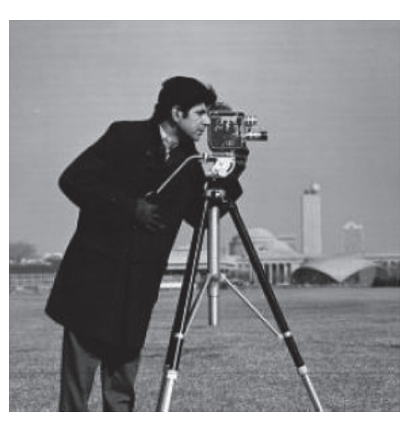

(b)

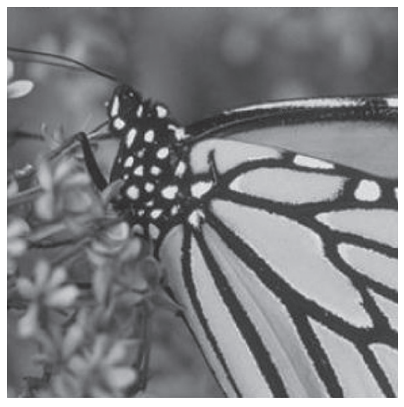

(e)

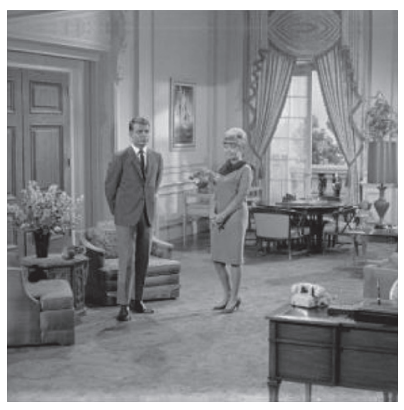

(h)

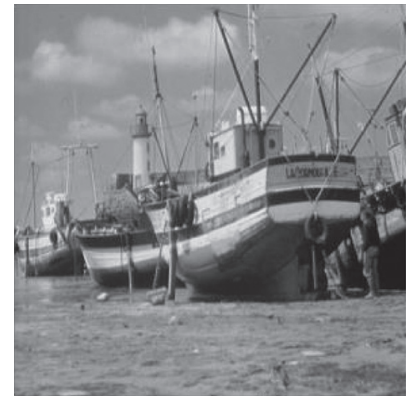

(c)

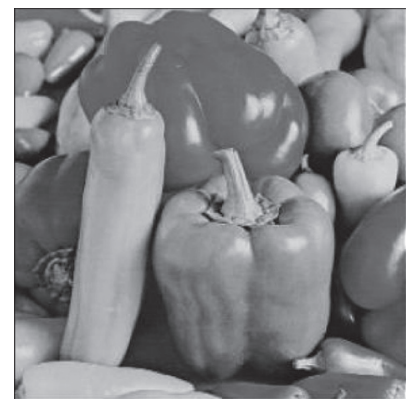

(f)

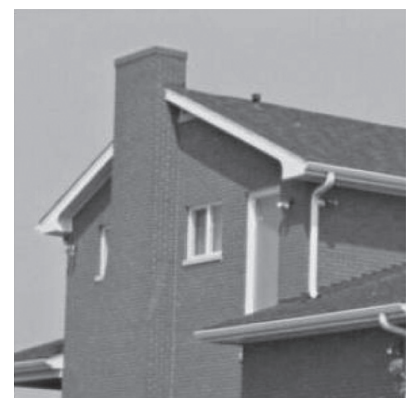

(i)

Figure 2: Test images for image restoration. 
TABLE 5: PSNR (dB) and FSIM results for $60 \%$ missing entries by different restoration methods.

\begin{tabular}{|c|c|c|c|c|c|}
\hline Image & BPFA & TNNR & TSLRA & WNNM-MC & NGRR \\
\hline Barbara & $31.36 / 0.94$ & $24.75 / 0.81$ & $33.17 / 0.97$ & $33.80 / 0.97$ & $34.27 / 0.98$ \\
\hline Couple & $30.56 / 0.96$ & $25.73 / 0.79$ & $30.02 / 0.95$ & $30.51 / 0.96$ & $30.47 / 0.96$ \\
\hline Boat & $30.23 / 0.95$ & $24.69 / 0.80$ & $30.01 / 0.95$ & $30.41 / 0.96$ & 30.52/0.95 \\
\hline Hill & $32.32 / 0.95$ & $27.45 / 0.83$ & $31.68 / 0.95$ & $32.42 / 0.96$ & $32.48 / 0.96$ \\
\hline Cameraman & $27.28 / 0.95$ & $22.95 / 0.79$ & $28.41 / 0.97$ & $28.43 / 0.97$ & 28.82/0.94 \\
\hline Peppers & $30.48 / \mathbf{0 . 9 6}$ & $25.07 / 0.78$ & $31.39 / 0.96$ & $29.81 / 0.95$ & $30.62 / 0.95$ \\
\hline Mandril & $30.50 / 0.94$ & $24.73 / 0.77$ & $30.51 / 0.94$ & $30.94 / 0.95$ & $31.10 / 0.95$ \\
\hline House & $36.17 / 0.97$ & $28.33 / 0.81$ & $37.45 / 0.98$ & $37.35 / \mathbf{0 . 9 8}$ & $37.41 / \mathbf{0 . 9 8}$ \\
\hline Montage & $27.87 / 0.95$ & $25.70 / 0.75$ & $28.29 / 0.96$ & $28.06 / 0.96$ & 29.18/0.97 \\
\hline Average & $30.75 / 0.95$ & $25.49 / 0.79$ & $31.21 / 0.96$ & $31.30 / 0.96$ & $31.65 / 0.96$ \\
\hline
\end{tabular}

TABLE 6: PSNR (dB) and FSIM results for 70\% missing entries by different restoration methods.

\begin{tabular}{|c|c|c|c|c|c|}
\hline Image & BPFA & TNNR & TSLRA & WNNM-MC & NGRR \\
\hline Barbara & $28.93 / 0.94$ & $26.68 / 0.73$ & $31.24 / 0.96$ & $31.63 / 0.96$ & $32.08 / 0.96$ \\
\hline Couple & 28.33/0.91 & $23.72 / 0.73$ & $28.28 / 0.91$ & $28.30 / 0.90$ & $28.36 / 0.91$ \\
\hline Boat & $28.00 / 0.91$ & $22.93 / 0.72$ & $28.19 / 0.91$ & $28.33 / \mathbf{0 . 9 3}$ & $28.44 / 0.93$ \\
\hline Hill & $30.55 / 0.93$ & $25.45 / 0.79$ & $30.35 / 0.93$ & $30.69 / 0.94$ & $30.65 / 0.94$ \\
\hline Cameraman & $25.65 / 0.89$ & $21.10 / 0.71$ & $27.12 / \mathbf{0 . 9 1}$ & $26.28 / 0.90$ & $27.24 / 0.91$ \\
\hline Peppers & $26.10 / 0.91$ & $22.58 / 0.71$ & $27.65 / 0.93$ & $27.63 / 0.92$ & $28.30 / 0.93$ \\
\hline Mandril & $28.78 / 0.90$ & $22.81 / 0.72$ & $29.17 / 0.92$ & $29.17 / 0.93$ & $29.41 / 0.93$ \\
\hline House & $33.13 / 0.94$ & $25.90 / 0.75$ & $34.93 / 0.96$ & $35.56 / 0.96$ & $35.00 / \mathbf{0 . 9 6}$ \\
\hline Montage & $26.18 / 0.94$ & $22.98 / 0.70$ & $26.53 / 0.95$ & $25.38 / \mathbf{0 . 9 5}$ & $27.31 / 0.95$ \\
\hline Average & $28.41 / 0.92$ & $23.79 / 0.73$ & $29.27 / 0.93$ & $29.22 / 0.93$ & 29.64/0.94 \\
\hline
\end{tabular}

TABLE 7: PSNR (dB) and FSIM results for $80 \%$ missing entries by different restoration methods.

\begin{tabular}{|c|c|c|c|c|c|}
\hline Image & BPFA & TNNR & TSLRA & WNNM-MC & NGRR \\
\hline Barbara & $26.98 / 0.91$ & $20.40 / 0.65$ & $28.98 / 0.93$ & $29.11 / 0.93$ & $29.63 / 0.94$ \\
\hline Couple & $26.05 / 0.91$ & $21.64 / 0.64$ & $26.28 / 0.92$ & $26.34 / 0.92$ & $26.40 / 0.92$ \\
\hline Boat & $25.99 / 0.87$ & $20.52 / 0.65$ & $26.29 / 0.93$ & $26.46 / 0.89$ & $26.60 / 0.89$ \\
\hline Hill & $28.47 / 0.89$ & $23.15 / 0.71$ & $28.47 / 0.89$ & $28.50 / \mathbf{0 . 9 0}$ & $28.58 / 0.90$ \\
\hline Cameraman & $23.91 / 0.87$ & $18.63 / 0.61$ & $24.59 / 0.89$ & $24.68 / 0.86$ & $25.31 / 0.88$ \\
\hline Peppers & $26.29 / 0.91$ & $20.47 / 0.64$ & $27.96 / \mathbf{0 . 9 3}$ & $27.61 / 0.92$ & 28.12/0.93 \\
\hline Mandril & $26.75 / 0.88$ & $20.37 / 0.64$ & $27.15 / 0.88$ & $27.12 / 0.89$ & 27.32/0.89 \\
\hline House & $30.18 / 0.91$ & $22.82 / 0.65$ & $31.71 / 0.93$ & $32.54 / 0.95$ & $32.23 / 0.94$ \\
\hline Montage & $24.43 / 0.90$ & $20.25 / 0.60$ & $24.96 / 0.93$ & $22.54 / 0.92$ & $24.40 / \mathbf{0 . 9 3}$ \\
\hline Average & $26.56 / 0.89$ & $20.92 / 0.64$ & $27.38 / 0.91$ & $27.21 / 0.91$ & $27.62 / 0.91$ \\
\hline
\end{tabular}

we try to recover images with $60 \%, 70 \%$, and $80 \%$ missing entries, respectively. PSNR and FSIM values of restored images by different methods are reported in Tables 5-7. Meanwhile, in Figures 3 and 4, image restoration results are also visually shown using different methods for Barbara and Peppers images with random loss of $80 \%$ pixels.

First, from Tables 5-7, one can see that PSNR $(\mathrm{dB})$ and FSIM results by the proposed method exceed those of other methods on the whole, which verifies the higher performance of NGRR in the restoration of missing data. Both TSLRA and WNNM-MC are also excellent image restoration methods in the case of severe data loss from the view of quantitative evaluation.

Secondly, in Figures 3 and 4, a visual interpretation of image restoration results by different methods can be conducted. The BPFA method produces blurry edges with lost textures and artifacts near edges in restored images. The TNNR method presents wrong linear textures due to its deficiency in restoration of fine textures and details. The TSLRA, WNNM-MC, and proposed NGRR methods obtain relatively better results in the recovery of image structures, textures, and details. Through careful observation, one can see that the NGRR method produces fewer artifacts than that of the WNNM-MC method and relatively sharper edges than that of the TSLRA method in image results. This observation also verifies better ability of sparse representation and information retrieval of the proposed method with the gradual regularization and the nonlocal low rank minimization. Meanwhile, it is obvious that the results by the methods with low rank approximation of whole image 


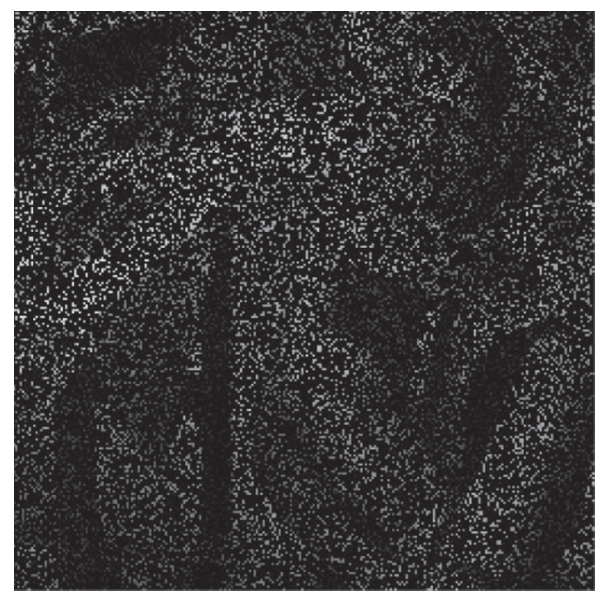

(a)

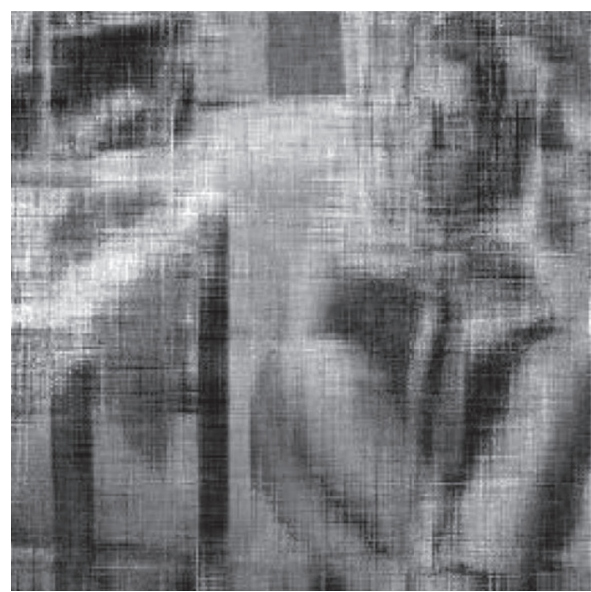

(c)

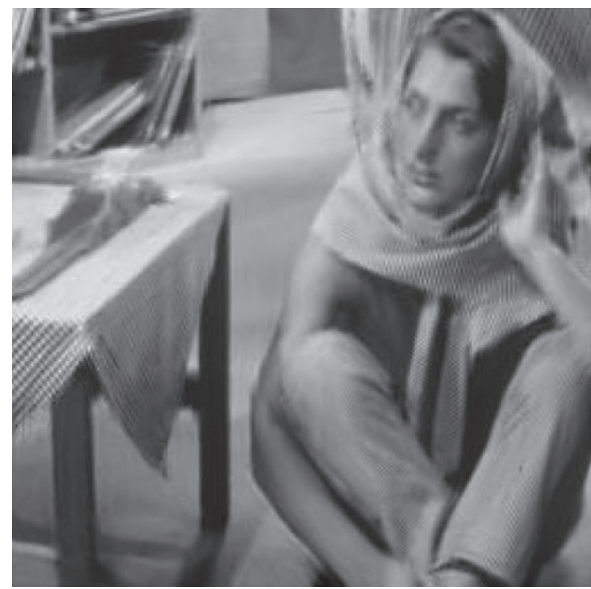

(e)

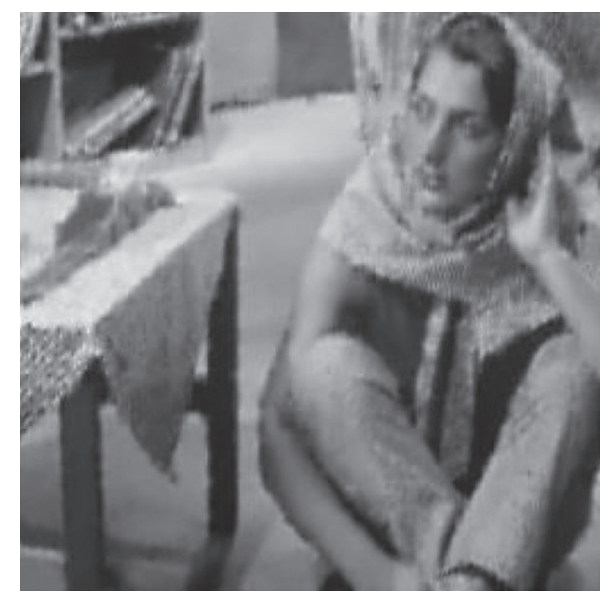

(b)

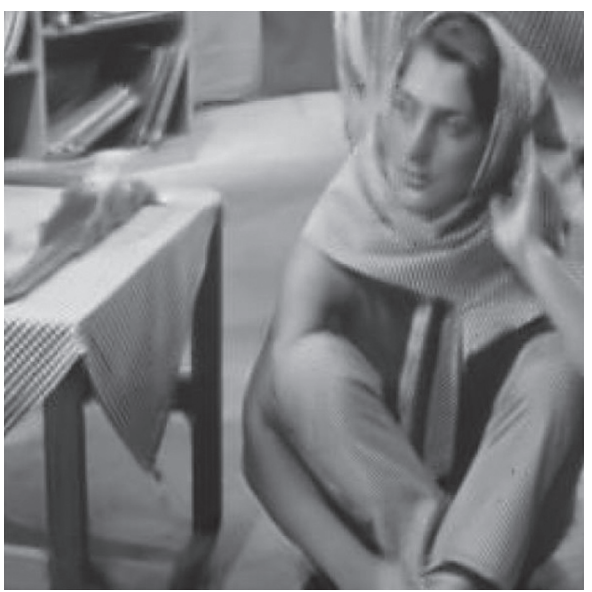

(d)

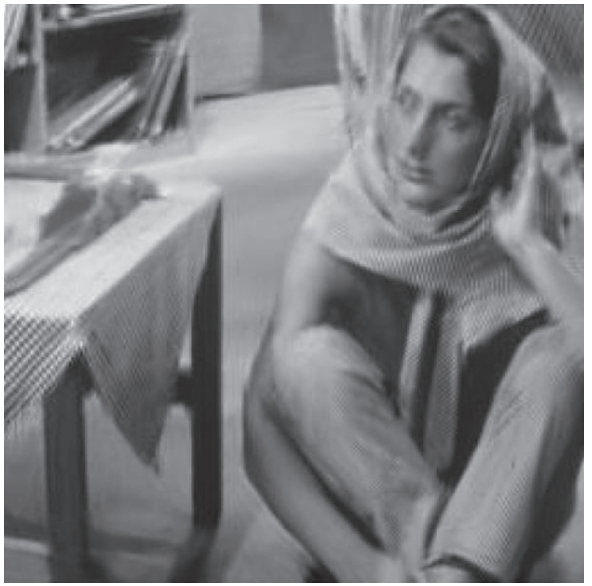

(f)

Figure 3: Comparison of restored Barbara images with $80 \%$ missing entries (from top-left to bottom-right): damaged image, results by BPFA, TNNR, TSLRA, WNNM-MC and NGRR methods, repectively.

matrix are not ideal in the task of randomly missing pixel recovery.

Finally, we discuss the parameter setting in the proposed method in image restoration. Our method includes three main parameters: block size $W$, number of similar blocks $N_{i}$, and iteration times $T$. In order to evaluate the effect of $W$ on image recovery, we test ten images with randomly missing pixels in experiments on natural gray image by adopting different $W$ while fixing other parameters. In Figure 5 the best result is obtained with $W=24$. In addition, in above 


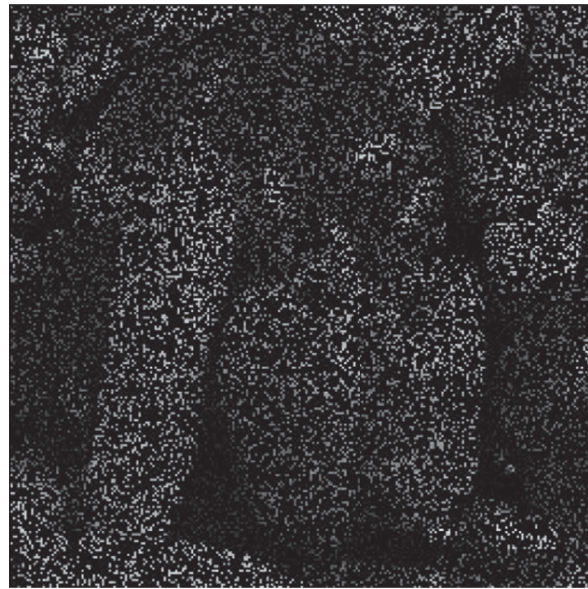

(a)

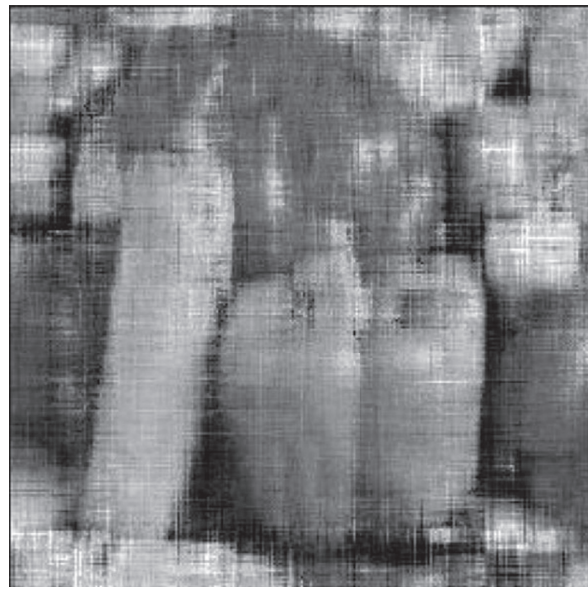

(c)

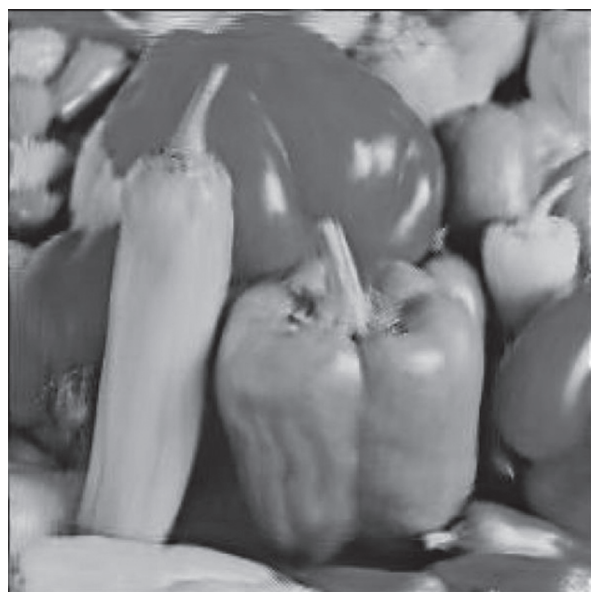

(e)

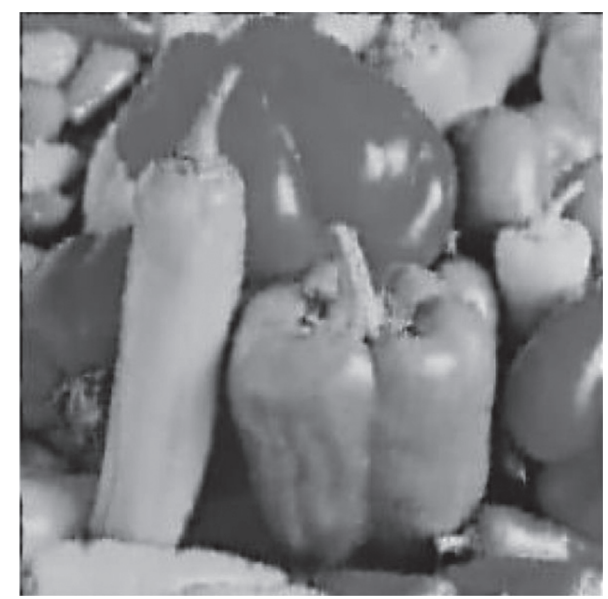

(b)

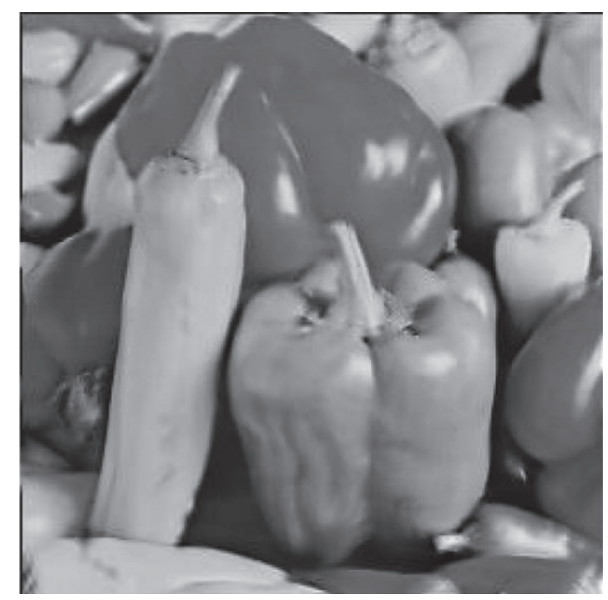

(d)

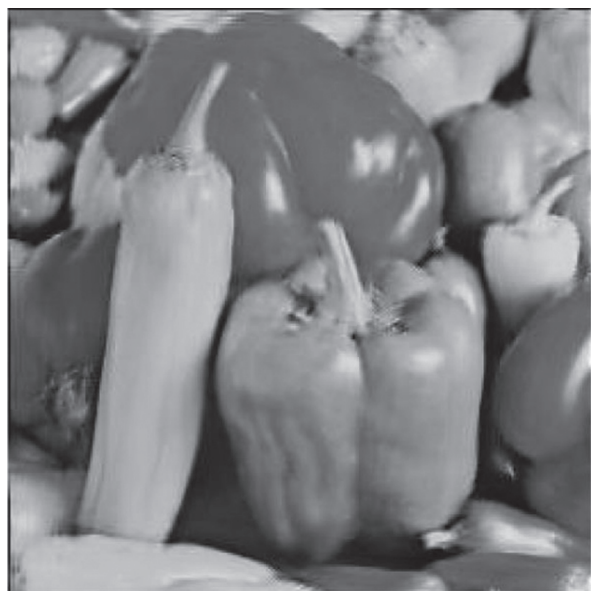

(f)

Figure 4: Comparison of restored Peppers images with $80 \%$ missing entries (from top-left to bottom-right): damaged image, results by BPFA, TNNR, TSLRA, WNNM-MC and NGRR methods, repectively.

experiments on natural images, the parameter setting in the proposed method includes $N_{i}=36$ for smooth block, $N_{i}=$ 48 for textured block, and $T=4$. For the sake of simplicity, Euclidean distance is used to measure the similarity between two image blocks.
All methods implemented in MATLAB are run on a laptop with Intel Core i3 CPU and 8 GB RAM. The average running time of BPFA, TNNR, TSLRA, WNNM-MC, and NGRR methods are 10.1, 2.2, 6.5, 12.7, and 13.8 minutes, respectively. 


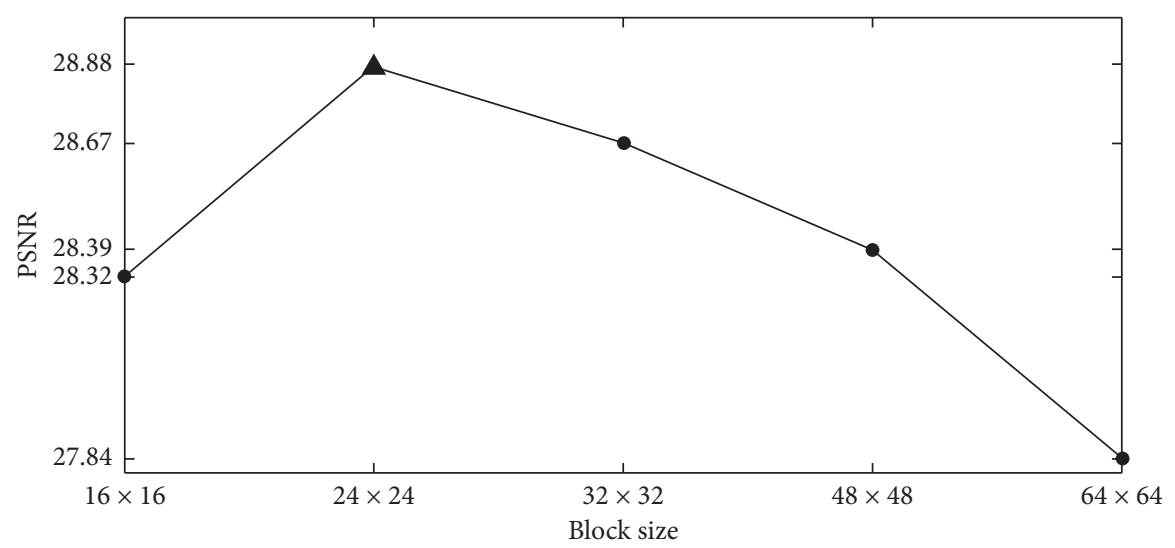

Figure 5: Mean values of PSNR results for different block sizes in restoration of test images.

\section{Conclusion}

An important need in computer vision is to use advanced image restoration technology to retrieve lost visual information from damaged image. In this paper, we propose a nonconvex minimization model with gradual reweighted regularization to restore missing pixels in digital image, making use of priors of low rank, nonlocal self-similarity and sparsity-promoting regularization. An alternation direction method of multipliers algorithm is used to iteratively solve above minimization problem. Experiments on simulated and real images have verified the effectiveness of the proposed algorithm. In future work, we will develop automatic detection algorithm of missing pixels of image and will combine the proposed method with exemplar synthesis for image restoration of big missing areas.

\section{Data Availability}

All test images can be downloaded from http://www.cs.tut.fi/ $\sim$ foi/GCF-BM3D/.

\section{Conflicts of Interest}

The authors declare that they have no conflicts of interest.

\section{Acknowledgments}

The research has been supported in part by the National Natural Science Foundation of China (61671276, 11971269), the Natural Science Foundation of Shandong Province of China (ZR2019MF045), and the Social Science Planning Research Project of Shandong Province of China. The author would like to thank Shujun Fu and his research group for their help in the preparation of this manuscript and MATLAB implementation.

\section{References}

[1] F. Stanco, S. Battiato, and G. Gallo, Digital Imaging for Cultural Heritage Preservation: Analysis, Restoration, and Reconstruction of Ancient Artworks, CRC Press, Boca Raton, FL, USA, 2011.
[2] F. Wang, "Comparative study on digital image enhancement for virtual restoration of mural painting," International Journal of Engineering and Technical Research, vol. 7, no. 12, pp. 137-140, 2017.

[3] M. Jmal, W. Souidene, and R. Attia, "Efficient cultural heritage image restoration with nonuniform illumination enhancement," Journal of Electronic Imaging, vol. 26, no. 1, Article ID 011020, 2017.

[4] M. Bertalmio, G. Sapiro, V. Caselles, and C. Ballester, "Image inpainting," in Proceedings of ACM SIGGRAPH, pp. 417-424, New Orleans, LA, USA, July 2000.

[5] T. F. Chan and J. Shen, "Mathematical models for local nontexture inpaintings," SIAM Journal on Applied Mathematics, vol. 62, no. 3, pp. 1019-1043, 2002.

[6] M. Bertalmio, L. Vese, G. Sapiro, and S. Osher, "Simultaneous structure and texture image inpainting," IEEE Transactions on Image Processing, vol. 12, no. 8, pp. 882-889, 2003.

[7] C. Guillemot and O. Le Meur, "Image inpainting: overview and recent advances," IEEE Signal Processing Magazine, vol. 31, no. 1, pp. 127-144, 2014.

[8] S.-J. Fu and Q.-Q. Ruan, "A local nontexture image inpainting and denoising based on nonlinear PDEs," in Proceedings of the 7th International Conference on Signal Processing, 2004. Proceedings. ICSP '04. 2004, vol. 2, pp. 1029-1032, Beijing, China, September 2004.

[9] K. Zhang, W. Zuo, S. Gu, and L. Zhang, "Learning deep CNN denoiser prior for image restoration," in Proceedings of IEEE Conference on Computer Vision and Pattern Recognition, pp. 3929-3938, Honolulu, HI, USA, July 2017.

[10] F. Wang, "A study of digital image enhancement for cultural relic restoration," International Journal of Engineering and Technical Research, vol. 7, no. 11, pp. 41-44, 2017.

[11] R. C. Gonzalez and R. E. Woods, Digital Image Processing, Prentice-Hall, New York, NY, USA, 2nd edition, 2004.

[12] T. F. Chan and J. Shen, "Nontexture inpainting by curvaturedriven diffusions," Journal of Visual Communication and Image Representation, vol. 12, no. 4, pp. 436-449, 2001.

[13] A. Criminisi, P. Perez, and K. Toyama, "Region filling and object removal by exemplar-based image inpainting," IEEE Transactions on Image Processing, vol. 13, no. 9, pp. 12001212, 2004.

[14] H. Zhang, Y. Jin, and Y. Wu, "Image completion by a fast and adaptive exemplar-based image inpainting," in Proceedings of the International Conference on Computer Application and System Modeling (ICCASM 2010), vol. 3, pp. 115-119, Taiyuan, China, October 2010. 
[15] K. A. Vahid and F. Yaghmaee, "Introducing a new fast exemplar-based inpainting algorithm," in Proceedings of the 2014 22nd Iranian Conference on Electrical Engineering (ICEE), pp. 874-878, Tehran, Iran, May 2014.

[16] A. C. Kokaram, "On missing data treatment for degraded video and film archives: a survey and a new Bayesian approach," IEEE Transactions on Image Processing, vol. 13, no. 3, pp. 397-415, 2004.

[17] M. Elad, J.-L. Starck, P. Querre, and D. L. Donoho, "Simultaneous cartoon and texture image inpainting using morphological component analysis (MCA)," Applied and Computational Harmonic Analysis, vol. 19, no. 3, pp. 340-358, 2005.

[18] J. C. Emmanuel and B. Recht, "Exact matrix completion via convex optimization," Foundations of Computational Mathematics, vol. 9, no. 6, pp. 717-772, 2009.

[19] H. Ji, S. Huang, Z. Shen, and Y. Xu, "Robust video restoration by joint sparse and low rank matrix approximation," SIAM Journal on Imaging Sciences, vol. 4, no. 4, pp. 1122-1142, 2011.

[20] W. Dong, G. Shi, and X. Li, "Nonlocal image restoration with bilateral variance estimation: a low-rank approach," IEEE Transactions on Image Processing, vol. 22, no. 2, pp. 700-711, 2013.

[21] F. Li and T. Zeng, "A universal variational framework for sparsity-based image inpainting," IEEE Transactions on Image Processing, vol. 23, no. 10, pp. 4242-4254, 2014.

[22] L. Ma and T. Zeng, "Image deblurring via total variation based structured sparse model selection," Journal of Scientific Computing, vol. 67, no. 1, pp. 1-19, 2016.

[23] L. Ma, L. Xu, and T. Zeng, "Low rank prior and total variation regularization for image deblurring," Journal of Scientific Computing, vol. 70, no. 3, pp. 1336-1357, 2017.

[24] S. Gu, Q. Xie, D. Meng, W. Zuo, X. Feng, and L. Zhang, "Weighted nuclear norm minimization and its applications to low level vision," International Journal of Computer Vision, vol. 121, no. 2, pp. 183-208, 2017.

[25] H. Lu, Q. Liu, M. Zhang, Y. Wang, and X. Deng, "Gradientbased low rank method and its application in image inpainting," Multimedia Tools and Applications, vol. 77, no. 5, pp. 5969-5993, 2018.

[26] Q. Guo, S. Gao, X. Zhang, Y. Yin, and C. Zhang, "Patch-based image inpainting via two-stage low rank approximation," IEEE Transactions on Visualization and Computer Graphics, vol. 24, no. 6, pp. 2023-2036, 2018.

[27] J.-P. Lewis, "Texture synthesis for digital painting," $A C M$ SIGGRAPH Computer Graphics, vol. 18, no. 3, pp. 245-252, 1984.

[28] A. A. Efros and T. K. Leung, "Texture synthesis by nonparametric sampling," in Proceedings of IEEE International Conference on Computer Vision, pp. 1033-1038, Kerkyra, Greece, September 1999.

[29] M. Elad and M. Aharon, "Image denoising via sparse and redundant representations over learned dictionaries," IEEE Transactions on Image Processing, vol. 15, no. 12, pp. 3736$3745,2006$.

[30] E. Candes, Y. Plan, Matrix completion with noise," Proceedings of the IEEE, vol. 98, no. 6, pp. 925-936, 2010.

[31] J.-F. Cai, J. C. Emmanuel, and Z. Shen, "A singular value thresholding algorithm for matrix completion," SIAM Journal on Optimization, vol. 20, no. 4, pp. 1956-1982, 2010.

[32] D. L. Donoho, "Compressed sensing," IEEE Transactions on Information Theory, vol. 52, no. 4, pp. 1289-1306, 2006.
[33] Z. Lin, "A review on low-rank models in data analysis," Big Data and Information Analytics, vol. 1, no. 2-3, pp. 139-161, 2016.

[34] G. Liu, Z. Lin, S. Yan, J. Sun, Y. Yu, and Y. Ma, "Robust recovery of subspace structures by low-rank representation," IEEE Transactions on Pattern Analysis and Machine Intelligence, vol. 35, no. 1, pp. 171-184, 2013.

[35] L. Zhai, S. Fu, H. Lv, C. Zhang, and F. Wang, "Weighted Schatten p-norm minimization for 3D magnetic resonance images denoising," Brain Research Bulletin, vol. 142, pp. 270-280, 2018.

[36] H. Lu, S. Li, Q. Liu, and M. Zhang, "MF-LRTC: multi-filters guided low-rank tensor coding for image restoration," Neurocomputing, vol. 303, pp. 88-102, 2018.

[37] H. Chen, S. Fu, H. Wang et al., "Feature-oriented singular value shrinkage for optical coherence tomography image," Optics and Lasers in Engineering, vol. 114, pp. 111-120, 2019.

[38] E. Candes, M. B. Wakin, and S. P. Boyd, "Enhancing sparsity by reweighted $l_{1}$ minimization," Journal of Fourier Analysis and Applications, vol. 14, no. 5-6, pp. 877-905, 2008.

[39] R. Chartrand and W. Yin, "Iteratively reweighted algorithms for compressive sensing," in Proceedings of the IEEE International Conference on Acoustics, Speech and Signal Processing, pp. 3869-3872, IEEE, Las Vegas, NV, USA, MarchApril 2008.

[40] S. Boyd, N. Parikh, E. Chu, P. Borja, and J. Eckstein, "Distributed optimization and statistical learning via the alternating direction method of multipliers," Foundations and Trends in Machine Learning, vol. 3, no. 1, pp. 1-122, 2011.

[41] A. Buades, B. Coll, and J.-M. Morel, "A non-local algorithm for image denoising," in Proceedings of IEEE Conference on Computer Vision and Pattern Recognition, vol. 2, IEEE, San Diego, CA, USA, pp. 60-65, June 2005.

[42] K. Dabov, A. Foi, V. Katkovnik, and K. Egiazarian, "Image denoising by sparse 3-D transform-domain collaborative filtering," IEEE Transactions on Image Processing, vol. 16, no. 8, pp. 2080-2095, 2007.

[43] Z. Zha, X. Zhang, Y. Wu et al., "Non-convex weighted $\ell_{\mathrm{p}}$ nuclear norm based ADMM framework for image restoration," Neurocomputing, vol. 311, pp. 209-224, 2018.

[44] H. Chen, S. Fu, H. Wang, H. Lv, and C. Zhang, "Speckle attenuation by adaptive singular value shrinking with generalized likelihood matching in optical coherence tomography," Journal of Biomedical Optics, vol. 23, no. 3, Article ID 036014, 2018.

[45] M. Zhou, H. Chen, J. Paisley et al., "Nonparametric Bayesian dictionary learning for analysis of noisy and incomplete images," IEEE Transactions on Image Processing, vol. 21, no. 1, pp. 130-144, 2012.

[46] Y. Hu, D. Zhang, J. Ye, X. Li, and X. He, "Fast and accurate matrix completion via truncated nuclear norm regularization," IEEE Transactions on Pattern Analysis and Machine Intelligence, vol. 35, no. 9, pp. 2117-2130, 2013.

[47] L. Zhang, L. Zhang, X. Mou, and D. Zhang, "FSIM: a feature similarity index for image quality assessment," IEEE Transactions on Image Processing, vol. 20, no. 8, pp. 2378-2386, 2011. 